\title{
Management strategies of potentially resectable colorectal liver metastases in a cohort of Bangladeshi patients
}

\author{
Rabbi H ${ }^{\mathrm{a}}$, Rahid MM ${ }^{\mathrm{a}}$, Hakim HAN ${ }^{\mathrm{b}}$, Chowdhury $\mathrm{AQ}^{\mathrm{b}}$, Islam A ${ }^{\mathrm{b}}$, Ahmed $\mathrm{AHMT}^{\mathrm{a}}$,

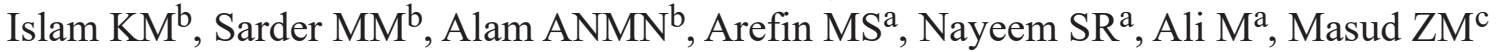

\begin{abstract}
Background: Colorectal cancer with liver metastases (CRLM) is stage IV disease. Only 60\% patients present with palpable liver or a liver mass and at laparotomy $80 \%$ hepatic metastases can be detected. Synchronous CRLM (SCRRLM) is indicative of poor prognosis than metachronous (MCRLM) counterpart. Only 13-15\% of SCLM are eligible for curative resection. Surgical intervention offers long term cure with overall survival in 37-58\% patients. This study was designed to validate different approaches of management to patients with CRLM in Bangladeshi patients.

Methods: In this prospective observational study, we observed different management approaches in 41 Bangladeshi individuals with CRLM from January 2010 to January 2018 in different tertiary care hospitals of Dhaka. They were thoroughly evaluated and prepared for surgical resection. After detection both synchronous and metachronous CRLM, patients were treated surgically with colonic resection and liver resection with simultaneous approach, lesion first approach, liver first approach. Intraoperative ultrasound was valuable in localization of liver lesions. Patients were followed up for a minimum 6 months to maximum period of 61 months.

Results: The study included 41 patients between ages of 21 to 70 years, of them $22(53.65 \%)$ males and 19 (46.34\%) were female. Among them, 19 patients (46.34\%) had synchronous lesion and 22 (53.66\%) had metachronous lesion. Neoadjuvant therapy was given in $9(21.95 \%)$ patients. All the patients received adjuvant therapy. Multiple metastetectomy was done in 31 (75.60\%) patients. In our series, following margin negative hepatic resection, 14 (34.14\%) patients survived 3 years and $3(7.31 \%)$ patients survived 5 years.

Conclusion: CRLM signifies an advanced disease at presentation. Hepatic resection following resection of colorectal primary is curative. Simultaneous liver with colonic resection is safe and effective in cases of small hepatic metastases.
\end{abstract}

Key wards: colon cancer, colorectal liver metastases, liver metastases, synchronous colon cancer.

(BIRDEM Med J 2019; 9(3): 207-212)

\section{Author information}

a. Hashim Rabbi, Md. Mamunur Rashid, AHM Tanvir Ahmed, Mirza Shamsul Arefin, Sarder Rizwan Nayeem, Mohammad Ali, Department of Hepato-Biliary-Pancreatic Surgery, BIRDEM General Hospital, Dhaka-1000, Bangladesh.

b. H A Nazmul Hakim, Ajmal Quader Chowdhury, Aminul Islam, Kazi Mazharul Islam, Mahmud Mohammad Sarder, ANM Nure Alam, Department of Surgery, Dhaka Medical College Hospital, Dhaka, Bangladesh.

c. Zafor Mohammad Masud, Department of Oncology, Bangladesh Medical College Hospital, Dhaka, Bangladesh.

Address of correspondence: Dr. Hashim Rabbi, MBBS, FCPS, MRCS Ed, MRCPS (Glasg.), Associate Professor, Department of Hepato-Biliary-Pancreatic Surgery [HBPS], Room 710, BIRDEM General Hospital, 122 Kazi Nazrul Islam Avenue, Shahbag, Dhaka1000, Bangladesh. Email: hrabbi72@gmail.com

Received: January 27, 2019

Accepted: July 31, 2019

\section{Introduction}

Metastatic liver tumor is 20 times more common than primary. Colorectal cancer (CRC) is a very common malignancy worldwide and development of liver metastases, both synchronous or metachronous (CRLM), is not a rare event. Among patients with metastatic colorectal cancer, up to $77 \%$ have a liveronly disease and approximately $10 \%-20 \%$ of patients with colorectal liver metastases are considered resectable at the time of diagnosis. Surgical resection of liver metastases remains the paramount treatment option associated with a survival plateau. In addition a 20\%-25\% have long-term survival. ${ }^{1,2}$ CRLM is stage IV disease. More than $50 \%$ of patients with CRC will 
develop liver metastases during their lifespan. Synchronous colorectal liver metastases (SCRLM) are metastatic liver tumors, detected at initial presentation of primary CRC and metachronous colorectal liver metastases (MCRLM) are liver metastases that develop after resection of primary CRC. Hepatic resections for CRLM following resection of colorectal primary is a standard procedure. However, simultaneous liver with colonic resection is a safe and effective option in patients with small liver metastases. This study intended to further validate the different approaches to Bangladeshi patients with CRLM.

\section{Methods}

In this prospective observational study, we observed the outcome of different approaches in 41 consecutive Bangladeshi patients with CRLM, carried out from January 2010 to January 2018, managed by same surgical team at BIRDEM General Hospital, Dhaka Medical College Hospital and BRB hospitals Ltd. of Bangladesh. The patients were segregated into SCRLM and MCRLM, according to presentation. They were thoroughly evaluated. Apart from colonoscopy, ultra sonogram (USG), computed tomography scan (CTscan), magnetic resonance imaging (MRI), staging laparoscopy and positron emission tomography (PET CT-scan) was done where appropriate. After detection, patients were managed surgically with colonic and liver resection with simultaneous approach, primary or lesion first approach and liver first approach according to presentation. Intraoperative USG was a valuable adjunct in tumor localization. Neoadjuvant therapy was offered to 9 patients; however, all the patients received adjuvant chemotherapy. They were followed up from minimum 6 months to maximum period of 61 months. Chemotherapeutic agents, targeted therapy against vascular endothelial growth factor (VEGF), epidermal growth factor (EGF) were given according to protocol. In order to improve efficacy and response rate, these agents were often used in combinations where applicable. All CRLM patients with complete medical records, histopathology study and imaging findings to evaluate tumor extension were included in this study. Patients with medical records not complying with study variables, tumors treated with radiofrequency ablation, microwave ablation, tumors associated with inflammatory bowel disease were excluded.

\section{Follow-up after resection}

Patients, following hepatic and/or colorectal resection were monitored following guidelines to identify early recurrence. Patients underwent physical examination, serum carcino-embryonic antigen (CEA) level, chest Xray, CT-scan and PET-scan in selected cases, every 3 to 4 months for the first two years and then every 6 months for the following five years.

\section{Data collection and analysis}

Findings of observation were recorded on prescribed data collection from. After collection, data editing and clearing was done manually and prepared for data entry and calculated by computer based software.

\section{Results}

The study comprised consecutively selected 41 patients diagnosed as CRLM. Surgeries were performed according to mode of presentation following the protocol. Patients were between 21 and 70 years of age, $34(82.92 \%)$ patients were between $3^{\text {rd }}$ and $5^{\text {th }}$ decades of life, only $7(17.07 \%)$ patients were in $2^{\text {nd }}$ and $3^{\text {rd }}$ decades of life with highest $13(31.70 \%)$ patients between 41 and 50 years age group (Figure 1). We found, $22(53.65 \%)$ patients were male and 19(46.34\%) patients were female. Study showed a slight male predominance and presented at an earlier age than females.

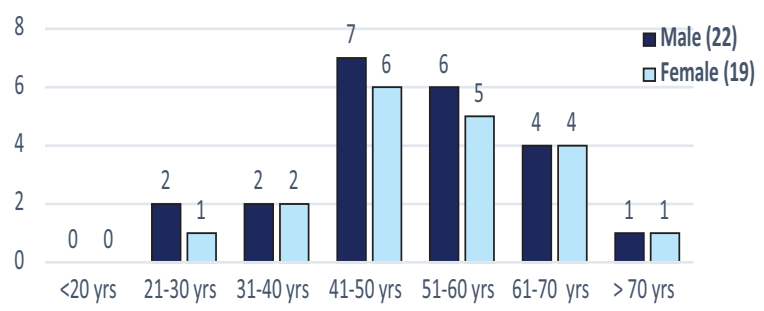

Figure 1 Age distribution of the study participants $(\mathrm{N}=41)$

In our series, 19 (46.34\%) patients had SCRLM and 22 (53.66\%) cases with MCRLM. Thirty four patients had primary site in the right colon and 6 patients in left colon and with only one patient in rectum. Fifteen $(36.58 \%)$ patients with caecal carcinoma, 9 patients with carcinoma ascending colon, 6 patients right colic flexure and 4 patients right transverse colon (Figure 2). However, 9 patients had their metastases in right lobe of liver, 13 patients in left lobe and 19 patients had bilateral disease. Simultaneous resection of primary CRC with hepatic resection was performed in 16 cases. Primary first approach was done in two patients and liver first in one. Thirteen patients under went right hemicolectomy with hepatic resection of CRLM (Table I). 


\begin{tabular}{|c|c|c|c|}
\hline & SCRLM & MCRLM & Total \\
\hline \multicolumn{4}{|l|}{ Gender } \\
\hline - Male & $12(29.26 \%)$ & $10(24.39 \%)$ & $22(53.65 \%)$ \\
\hline - Female & $7(17.07 \%)$ & $12(29.26 \%)$ & $19(46.34 \%)$ \\
\hline Mode of presentation & $19(46.34 \%)$ & $22(53.65 \%)$ & \\
\hline \multicolumn{4}{|l|}{ Primary site } \\
\hline - Rt. Colon & $13(31.70 \%)$ & $20(48.78 \%)$ & $33(80.48 \%)$ \\
\hline - $\quad$ Lt. Colon & $1(2.43 \%)$ & $6(14.63 \%)$ & $7(17.07 \%)$ \\
\hline - $\quad$ Rectum \& Anal canal & 0 & $1(2.43 \%)$ & $1(2.43 \%)$ \\
\hline \multicolumn{4}{|l|}{ Site at liver } \\
\hline - $\quad$ Rt. Lobe & $8(19.51 \%)$ & $1(2.43 \%)$ & $9(21.95 \%)$ \\
\hline - $\quad$ Lt. Lobe & $7(17.07 \%)$ & $6(14.63 \%)$ & $13(31.70 \%)$ \\
\hline - $\quad$ Both Lobes & $12(29.26 \%)$ & $7(17.07 \%)$ & $19(46.34 \%)$ \\
\hline \multicolumn{4}{|l|}{ Surgical approaches } \\
\hline - Simultaneous approach & $16(39.02 \%)$ & & \\
\hline - $\quad$ Primary/Sequential approach & $2(4.87 \%)$ & & $19(46.34 \%)$ \\
\hline - $\quad$ Liver first approach & $1(2.43 \%)$ & & \\
\hline \multicolumn{4}{|l|}{ Type of liver resections } \\
\hline - Multiple metastetectomy & $16(39.02 \%)$ & $15(36.58 \%)$ & $31(75.60 \%)$ \\
\hline$>3$ Lesions & $5(12.19 \%)$ & $7(17.07 \%)$ & \\
\hline$<3$ Lesions & $10(24.39 \%)$ & $9(21.95 \%)$ & \\
\hline Lt. lateralhep.segmentectomy & $3(7.31 \%)$ & $5(12.19 \%)$ & $8(19.51 \%)$ \\
\hline Rt. hepatectomy & $1(2.43 \%)$ & $1(2.43 \%)$ & \\
\hline - Lt. hepatectomy & $1(2.43 \%)$ & $1(2.43 \%)$ & \\
\hline \multicolumn{4}{|l|}{ Survival } \\
\hline - 3 years & $10(24.39 \%)$ & $4(9.75 \%)$ & $14(34.14 \%)$ \\
\hline - $\quad 5$ years & $2(4.87 \%)$ & $3(7.31 \%)$ & $5(12.19 \%)$ \\
\hline
\end{tabular}

$[R t .=R i g h t, L t .=$ Left, CRLM: Colorectal liver metastases, SCRLM: Synchronous colorectal liver metastases, MCRLM: Metachronous colorectal liver metastases]

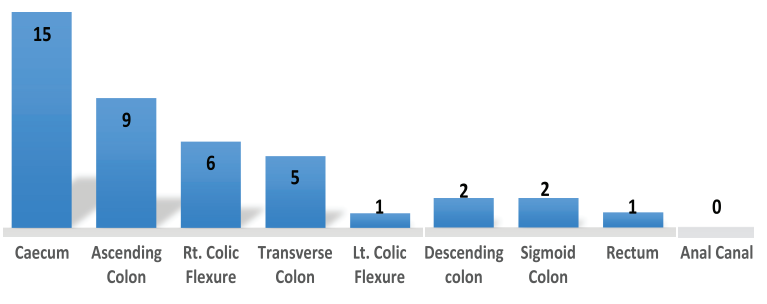

Figure 2 Distribution of primary CRC $(\mathrm{N}=41)$

Multiple metastetectomy was performed in 31 (75.60\%) patients. Metastetectomy was done for more than three lesions in $12(29.26 \%)$ patients and in $19(46.34 \%)$ patients for three or less lesions. Multiple metastetectomy was performed with varying diameter lesions with largest one $7 \mathrm{~cm}$. Segmental resection was preferred in larger lesion and multiple metastases with involved hepatic segment or lobe.

Table II Postoperative complications $(\mathrm{N}=7)$

\begin{tabular}{lcc}
\hline Complications & No. & $\%$ \\
\hline Wound Infection & 7 & 17.07 \\
Burst abdomen & 2 & 4.87 \\
Intra-abdominal sepsis & 4 & 9.75 \\
Hemorrhage & 2 & 4.87 \\
Anastomotic leakage & 1 & 2.43 \\
Biliary Leakage & 0 & 0 \\
Basal atelectasis & 3 & 7.31 \\
Paralytic ileus & 5 & 12.9 \\
\hline
\end{tabular}


Postoperative morbidity was noted in $7(17.07 \%)$ cases with no biliary leakage or immediate postoperative 30 days mortality. However, there was aminor colonic anastomotic leakage in our series, treated conservatively. Seven patients had wound infection and required wound care, one with methicillin-resistant Staphylococcus aureus (MRSA) positive strain was treated accordingly. Burst abdomen was noted in 2 patients who required immediate repair with tension suture. Among other complications, 3 (7.31\%) patients had basal atelectasis and 5 patients $(12.19 \%)$ developed paralytic ileus as they were with uncontrolled glycemic status for prolong period and required high doses of insulin (Table II). All patients received adjuvant chemotherapy and radiotherapy in 11 patients. They were treated by multidisciplinary team of specialists and followed up for a minimum 6 months to a maximum period of 61 months. However 6 patients $(14.63 \%)$ were dropped out during follow-up. In our series, for margin negative hepatic resection, the 3 years survival was $34.14 \%(n=14)$ patients and 5 years survival in was $7.31 \%(\mathrm{n}=3)$.

\section{Discussion}

CRLM is stage IV disease. Only $60 \%$ presents with palpable liver or a liver metastasis. Moreover, $80 \%$ hepatic metastases can be seen or felt during abdominal survey in laparotomy. Not a single hepatic segment is spared by CRLM. A quarter of patients with primary CRC presents with SCRLM and half of patients undergoing colonic resection eventually develop MCRLM. ${ }^{2,3}$ More than $50 \%$ of patients with CRC will develop liver metastases during their lifespan., 4 SCRLM are suggestive of poor prognosis than MCRLM. Only 13-15\% of SCLM are eligible curative resection, offers long term cure and survival in $37-58 \%$ patients. Approaches to these patients are centered on presentation. Our study included 41 patients of CRLM in different hospitals of Dhaka. Neoadjuvant therapy was offered to 9 patients and adjuvant chemotherapy to all. The series showed a slight male predominance and they presented at an earlier age. Synchronous lesion were less common than its metachronous counterpart. Stepwise haematogenous spread via portal circulation from the primary site to liver and from there to other organs provides an opportunity to prevent dissemination of tumor by direct interference of hepatic metastases. ${ }^{6,7}$ Evidence based on numerous retrospective and comparative studies indicate that hepatic resection is the only available treatment that allows long-term survival. ${ }^{8}$ Experiences with liver resection is associated with a $25 \%$ to $51 \%$ 5-year survival. ${ }^{9,10}$ Emerging strategies designed to increase the proportion of patients who are candidates for complete surgical resection. Despite improvements in chemotherapies and biological agents, survival is rarely longer than three years. 6,7 Neoadjuvant chemotherapy ${ }^{11}$, preoperative portal vein embolization ${ }^{12}$ and the two-stage resection approach 13 contribute to this aim.

Approaches are according to presentation, primary first or sequential approach, simultaneous or combined approach, reverse sequential or liver first approach, two staged hepatectomy and extreme liver surgery. The principle of hepatic resection in CRLM is not different from any other hepatic resections. Metastetectomy with healthy margin is the choice of surgical option. In young and normal healthy liver, removal of up to $80 \%$ of liver can be possible. In post chemotherapy or with mild fatty change up to $60-70 \%$ of liver can be possible. In chronic hepatitis, up to $50-60 \%$ hepatic resection is possible. The current criteria focus on what should be left after hepatic resection. Previous criteria for resection, such as the size, location, number of intrahepatic metastases and the presence of bilobar or extrahepatic disease have been largely abandoned. Now a days, the definition of resectability includes a complete resection with tumorfree surgical margins (R0 resection), sparing at least two liver segments. The main purpose of liver resection is to resect the tumor with a sufficient tumor-free margin, while preserving as much normal parenchyma as possible. ${ }^{12}$ Histologic pattern of tumor, bilateral or unilateral disease not impact outcome. More than 3 lesions, bilobar distributions, extrahepatic disease are no longer contraindications to hepatic resection. Whether lobectomy or segmentectomy or wedge resection, metastetectomy with healthy margin is the choice of surgical option. ${ }^{13,14}$ We have performed multiple metastetectomy in 31 patients with more than three metastetectomy in 12 patients, however less than three metastetectomy was done in 19 patients. Simultaneous resection of primary CRC with hepatic metastases was performed in 16 cases of SCRLM. Among them 13 cases under went right hemicolectomy with hepatic resection. However, primary first approach was done in two patients and liver first in one patient. Hepatic resection is performed with intention to cure, 
but $60 \%$ to $70 \%$ of patients develop local or distant relapse. ${ }^{15-17}$ Recurrence occurs equally at intrahepatic and extrahepatic sites; $80 \%$ of all recurrences occur within two years. The median survival of patients with recurrent disease is 8 to 10 months without any treatment. Repeat resection is possible in $10 \%$ to $15 \%$ cases and may achieve a five-year overall survival of $15-40 \%$ in selected individuals. A 10 -year disease-free survival is considered as cure. ${ }^{18-20}$

With improvement in imaging techniques, refinements in surgical techniques, progress in perioperative care, the postoperative mortality rate after hepatectomy has been reduced to $<3 \%$ and the five-year survival rate after resection of CRLM has reached 26\%-58\%. ${ }^{21-24}$ Besides, non-anatomical or wedge resection, removing a smaller volume of liver has reduced postoperative morbidity and mortality. Wedge resections in single rather than multiple lesions, incidence of positive resection margins was equivalent for both wedge resection and segmental resection $(8.3 \%)$ and the fiveyear survival was equivalent in both groups. ${ }^{25-}$ ${ }^{27}$ Evidence based numerous studies designate that hepatic resection is the only available treatment that allows long-term survival. The prognosis without hepatic resection with regional therapies, systemic chemotherapies and biological agents, median survival is 5-10 months and rarely approaches 3 years. However, survival after margin negative hepatic resection, the 5 year survivalis $40 \%$ and 10 year survivalis $20 \%$. $^{28,29}$ Five-year survivors with chemotherapy alone are anecdotal. ${ }^{30}$ Ensuing the standard protocol, they were treated and followed up by multi-disciplinary team of specialists and followed up accordingly. We found, in margin negative hepatic resection, the 3 year survival in $14(34.14 \%)$ patients and 5 years survival in 3 (7.31\%).

The study has few limitations, conducted with a small sample size and not representative of the whole country or region. More representative findings can be obtained from large sample size and in different tertiary level hospitals. We have $14.63 \%$ dropout in our series.

CRLM is an advanced disease but proper diagnosis, appropriate planning of surgical management with chemotherapy may appear curative, as was seen in this study.
Conflict of interest: Nothing to declare.

\section{References}

1. Remontet L, Estève J, Bouvier AM, Grosclaude P, Launoy G, Menegoz F, et al. Cancer incidence and mortality in France over the period 1978-2000. Rev Epidemiol Sante Publique 2003;51:3-30.

2. Evangelos P Misiakos, Nikolaos P. Current treatment for colorectal liver metastases; WorldJ Gastroenterol2011 September 28; 17(36): 4067-4075.

3. Benoist S, Brouquet A, Penna C, Julié C, El Hajjam M, Chagnon $\mathrm{S}$, et al. Complete response of colorectal liver metastases after chemotherapy: does it mean cure? J ClinOncol 2006; 24: 3939-3945

4. Shaw IM, Rees M, Welsh FK, Bygrave S, John TG. Repeat hepatic resection for recurrent colorectal liver metastases is associated with favourable long-term survival. Br J Surg2006; 93: 457-464.

5. Meriggi F, Bertocchi P, Zaniboni A. Management of potentially respectable colorectal cancer liver metastases. World J Gastrointest Surg 2013 May 27; 5(5): 138-145.

6. Cunningham D, Humblet Y, Siena S, Khayat D, Bleiberg H, Santoro A, et al. Cetuximab monotherapy and cetuximab plus irinotecan in irinotecan-refractory metastatic colorectal cancer. N Engl J Med 2004;351:337-345

7. Saltz LB, Cox JV, Blanke C, Rosen LS, Fehrenbacher L, Moore MJ, et al. Irinotecan plus fluorouracil and leucovorin for metastatic colorectal cancer. Irinotecan Study Group. N Engl J Med 2000;343:905-914.

8. Fong Y, Fortner J, Sun RL, Brennan MF, Blumgart LH. Clinical score for predicting recurrence after hepatic resection for metastatic colorectal cancer: analysis of 1001 consecutive cases. Ann Surg 1999;230:309-318.

9. Adam R, Pascal G, Azoulay D, Tanaka K, Castaing D, Bis-muth $H$. Liver resection for colorectal metastases: the third hepatectomy. Ann Surg2003; 238: 871-883.

10. Adam R, Avisar E, Ariche A, Giachetti S, Azoulay D, Castaing D. Five-year survival following hepatic resection after neoadjuvant therapy for nonresectable colorectal. Ann SurgOncol 2001;8:347-353

11. Schiergens TS, Rentsch M, Kasparek MS, Frenes K, Jauch KW, Thasler WE. Impact of Perioperative Allogeneic Red Blood Cell Transfusion on Recurrence and Overall Survival After Resection of Colorectal Liver Metastases. Dis Col Rec 2015:58(1):74-82.

12. Adam R, Laurent A, Azoulay D, Castaing D, Bismuth H. Twostage hepatectomy: A planned strategy to treat irresectable liver tumors. Ann Surg 2000;232:777-785.

13. Sharma S, Camci C, Jabbour N. Management of hepatic metastasis from colorectal cancers: an update. J HepatobiliaryPancreatSurg 2008;15:570-580. 
14. Berardi G, De Man M, Laurent S, Smeets P. Radiologic and pathologic response to neoadjuvant chemotherapy predicts survival in patients undergoing the liver-first approach for synchronous colorectal liver metastases. European J Surg Oncol 2018:44(7):1069-1077.

15. Tanaka K, Kumamoto T, Nojiri K. Impact of Postoperative Morbidity on Long-Term Survival After Resection for Colorectal Liver Metastases; Annals of Surgical Oncology 2016;23(Suppl. 5):929-937.

16. Pessaux P, Lermite E, Brehant O, Tuech JJ, Lorimier G, Ar-naud JP. Repeat hepatectomy for recurrent colorectal liver metastases. J SurgOncol2006; 93: 1-7.

17. Tomlinson JS, Jarnagin WR, DeMatteo RP, Fong Y, Kornprat $\mathrm{P}$, Gonen $\mathrm{M}$, et al. Actual 10-year survival after resection of colorectal liver metastases defines cure. J ClinOncol 2007;25:4575-4580.

18. Zorzi D, Mullen JT, Abdalla EK, Pawlik TM, Andres A, $\mathrm{Mu}$-ratore A, et al. Comparison between hepatic wedge resection and anatomic resection for colorectal liver metastases. J GastrointestSurg2006; 10: 86-94.

19. Small R, Lubezky N, Ben-Haim M. Current controversies in the surgical management of colorectal cancer metastases to the liver. Isr Med Assoc J2007; 9: 742-747

20. Abdalla EK, Vauthey JN, Ellis LM, Ellis V, Pollock R, Broglio $\mathrm{KR}$, et al. Recurrence and outcomes following hepatic resection, radiofrequency ablation, and combined resection/ ablation for colorectal liver metastases. Ann Surg 2004;239:818-825.

21. Tanaka K, Shimada H, Matsuo K, Nagano Y, Endo I, Sekido $\mathrm{H}$, Togo S. Outcome after simultaneous colorectal and he-patic resection for colorectal cancer with synchronous me-tastases. Surgery2004; 136: 650-659.
22. Khatri VP, Chee KG, Petrelli NJ. Modern multimodality approach to hepatic colorectal metastases: solutions and controversies. SurgOncol2007; 16: 71-83

23. Elias D, Liberale G, Vernerey D, Pocard M, Ducreux M, Boige $\mathrm{V}$, et al. Hepatic and extrahepatic colorectal metastases: when resectable, their localization does not matter, but their total number has a prognostic effect. Ann SurgOncol 2005;12:900909.

24. Pawlik TM, Scoggins CR, Zorzi D, Abdalla EK, Andres A, Eng C, et al. Effect of surgical margin status on survival and site of recurrence after hepatic resection for colorectal metastases. Ann Surg 2005;241:715-722.

25. Altendorf-Hofmann A, Scheele J. A critical review of the major indicators of prognosis after resection of hepatic metastases from colorectal carcinoma. SurgOncolClin N Am 2003;12:165-192, xi

26. Vauthey JN, Pawlik TM, Abdalla EK, Arens JF, Nemr RA, Wei SH, et al. Is extended hepatectomy for hepatobiliary malignancy justified? Ann Surg 2004;239:722-730.

27. Taylor R, Fong Y. Surgical treatment of hepatic metastases from colorectal cancer. In: Blumgart LH. Surgery of the liver, biliary tract, and pancreas. 4th Ed. Philadelphia, PAÿ Saunders Elsevier; 2007. pp. 1178-1194.

28. Jones OM, Rees M, John TG, Bygrave S, Plant G. Biopsy of resectable colorectal liver metastases causes tumour dissemination and adversely affects survival after liver resection. Br J Surg 2005;92:1165-1168.

29. Hao CY, Ji JF. Surgical treatment of liver metastases of colorectal cancer: Strategies and controversies in 2006. Eur J SurgOncol 2006;32:473-483

30. Yoshihiro M, Aloia TA, Brudvik K, Schwarz L, Vauthey J, Conrad C. Parenchymal-sparing Hepatectomy in Colorectal Liver Metastasis Improves Salvageability and Survival. Ann Surg 2016; 263(1):146-152. 